\title{
Observation of magnetic fragmentation in spin ice
}

\author{
S. Petitt ${ }^{1 \star}$, E. Lhotel ${ }^{2 \star}$, B. Canals ${ }^{2}$, M. Ciomaga Hatnean ${ }^{3}$, J. Ollivier ${ }^{4}$, H. Mutka ${ }^{4}$, E. Ressouche ${ }^{5}$,
} A. R. Wildes ${ }^{4}$, M. R. Lees ${ }^{3}$ and G. Balakrishnan ${ }^{3}$

Fractionalized excitations that emerge from a many-body system have revealed rich physics and concepts, from composite fermions in two-dimensional electron systems, revealed through the fractional quantum Hall effect ${ }^{1}$, to spinons in antiferromagnetic chains ${ }^{2}$ and, more recently, fractionalization of Dirac electrons in graphene ${ }^{3}$ and magnetic monopoles in spin ice ${ }^{4}$. Even more surprising is the fragmentation of the degrees of freedom themselves, leading to coexisting and a priori independent ground states. This puzzling phenomenon was recently put forward in the context of spin ice, in which the magnetic moment field can fragment, resulting in a dual ground state consisting of a fluctuating spin liquid, a so-called Coulomb phase $^{5}$, on top of a magnetic monopole crystal ${ }^{6}$. Here we show, by means of neutron scattering measurements, that such fragmentation occurs in the spin ice candidate $\mathrm{Nd}_{2} \mathrm{Zr}_{2} \mathrm{O}_{7}$. We observe the spectacular coexistence of an antiferromagnetic order induced by the monopole crystallization and a fluctuating state with ferromagnetic correlations. Experimentally, this fragmentation manifests itself through the superposition of magnetic Bragg peaks, characteristic of the ordered phase, and a pinch point pattern, characteristic of the Coulomb phase. These results highlight the relevance of the fragmentation concept to describe the physics of systems that are simultaneously ordered and fluctuating.

The physics of spin ice materials is intimately connected with the pyrochlore lattice, composed of corner-sharing tetrahedra. On the corners of these tetrahedra reside rare-earth magnetic moments $\mathbf{J}_{i}$, which, as a consequence of the strong crystal electric field, are constrained to point along their local trigonal axes $\mathbf{z}_{i}$, and behave like Ising spins. The magnetic interactions are composed of nearestneighbour exchange $\mathcal{J}$ and dipolar interactions between spins $i$ and $j$ separated by a distance $\mathbf{r}_{i j}$ (ref. 7):

$$
\mathcal{H}=\mathcal{J} \sum_{\langle i, j\rangle} \mathbf{J}_{i} \cdot \mathbf{J}_{j}+\mathcal{D} r_{\mathrm{nn}}^{3} \sum_{\langle i, j\rangle}\left[\frac{\mathbf{J}_{i} \cdot \mathbf{J}_{j}}{\left|\mathbf{r}_{i j}\right|^{3}}-\frac{3\left(\mathbf{J}_{i} \cdot \mathbf{r}_{i j}\right)\left(\mathbf{J}_{j} \cdot \mathbf{r}_{i j}\right)}{\left|\mathbf{r}_{i j}\right|^{5}}\right]
$$

where $\mathcal{D}=\mu_{\mathrm{o}}\left(g_{\mathrm{J}} \mu_{\mathrm{B}}\right)^{2} /\left(4 \pi r_{\mathrm{nn}}^{3}\right), \mu_{0}$ is the permeability of free space, $g_{\mathrm{J}}$ is the Landé factor of the magnetic moment, $\mu_{\mathrm{B}}$ is the Bohr magneton and $r_{\mathrm{nn}}$ is the nearest-neighbour distance between rareearth ions. The nearest-neighbour spin ice Hamiltonian is obtained by truncating the Hamiltonian (1), yielding:

$$
\mathcal{H}_{\mathrm{nn}}=\frac{-\mathcal{J}+5 \mathcal{D}}{3} \sum_{\langle i, j\rangle} J_{i}^{z} J_{j}^{z}
$$

When the effective interaction $\mathcal{J}_{\text {eff }}=(-\mathcal{J}+5 \mathcal{D}) / 3$ is positivethat is, when the dipolar term overcomes the antiferromagnetic exchange-a very unusual magnetic state develops, known as the spin ice state. The system remains in a highly correlated but disordered ground state where the local magnetization fulfils the so-called 'ice rule': each tetrahedron has two spins pointing in and two spins pointing out (see Fig. 1a), in close analogy with the rule which controls the hydrogen position in water ice ${ }^{8}$. The extensive degeneracy of this ground state results in a residual entropy at low temperature which is well approximated by the Pauling entropy for water ice ${ }^{9}$

Such highly degenerate states, where the organizing principle is dictated by a local constraint, belong to the class of Coulomb phases $^{5,10,11}$ : the constraint (the ice rule for spin ice) can be interpreted as a divergence-free condition of an emergent gauge field. This field has correlations that fall off with distance like the dipolar interaction ${ }^{12,13}$. In reciprocal space, this power-law character leads to bow-tie singularities, called pinch points, in the magnetic structure factor. They form a key experimental signature of the Coulomb phase physics. They have been observed by neutron diffraction in the spin ice materials $\mathrm{Ho}_{2} \mathrm{Ti}_{2} \mathrm{O}_{7}$ and $\mathrm{Dy}_{2} \mathrm{Ti}_{2} \mathrm{O}_{7}$, in excellent agreement with theoretical predictions ${ }^{14,15}$.

Classical excitations above the spin ice manifold are defects that locally violate the ice rule and so the divergence-free condition: by reversing the orientation of a moment, 'three in-one out' and 'one in-three out' configurations are created (see Fig. 1b). Considering the Ising spins as dumbbells with two opposite magnetic charges at their extremities, such defects result in a magnetic charge in the centre of the tetrahedron, called a magnetic monopole, that give rise to a non-zero divergence of the local magnetization ${ }^{4}$.

Recently, theoreticians have introduced the concept of magnetic moment fragmentation ${ }^{6}$, whereby the local magnetic moment field fragments into the sum of two parts, a divergence-full and a divergence-free part (see Fig. 1c): for example, a monopole in the spin configuration $m=\{1,1,1,-1\}$ on a tetrahedron can be written $m=1 / 2\{1,1,1,1\}+1 / 2\{1,1,1,-3\}$. In this decomposition, the first term carries the total magnetic charge of the monopole. If the monopoles organize as a crystal of alternating magnetic charges, the fragmentation leads to the superposition of an ordered 'all inall out' configuration (Fig. 1d) and of an emergent Coulomb phase associated with the divergence-free contribution (Fig. 1e).

This monopole crystallization occurs when the monopole density is high enough such that the Coulomb interaction between monopoles (which originates in the dipolar interaction between magnetic moments) is minimized through charge ordering, whereas the remaining fluctuating divergence-free part provides a gain in entropy.

The necessary conditions for an experimental realization of this physics are severe: in pyrochlore systems, the fragmentation is expected in the case of strong Ising anisotropy combined with effective ferromagnetic interactions, and for a specific ratio between the dipolar and exchange interactions to form the crystal

${ }^{1}$ LLB, CEA, CNRS, Université Paris-Saclay, CEA Saclay, 91191 Gif-sur-Yvette, France. ${ }^{2}$ Institut Néel, CNRS and Université Grenoble Alpes, F-38042 Grenoble, France. ${ }^{3}$ Department of Physics, University of Warwick, Coventry CV4 7AL, UK. ${ }^{4}$ Institut Laue Langevin, F-38042 Grenoble, France. ${ }^{5}$ INAC, CEA and Université Grenoble Alpes, CEA Grenoble, F-38054 Grenoble, France. *e-mail: sylvain.petit@cea.fr; elsa.Ihotel@neel.cnrs.fr 

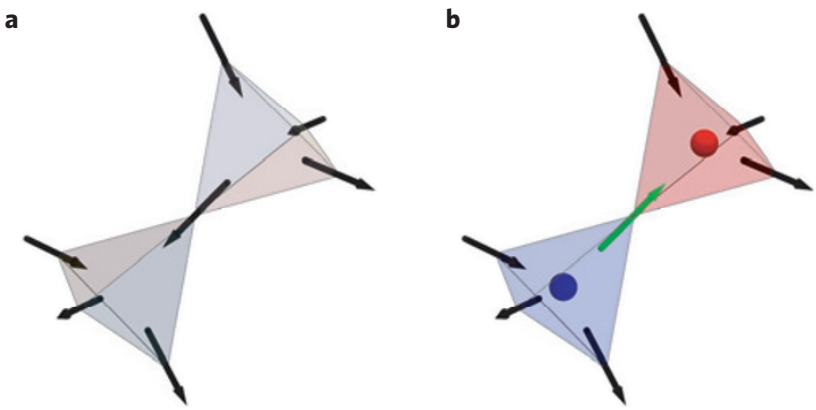

c

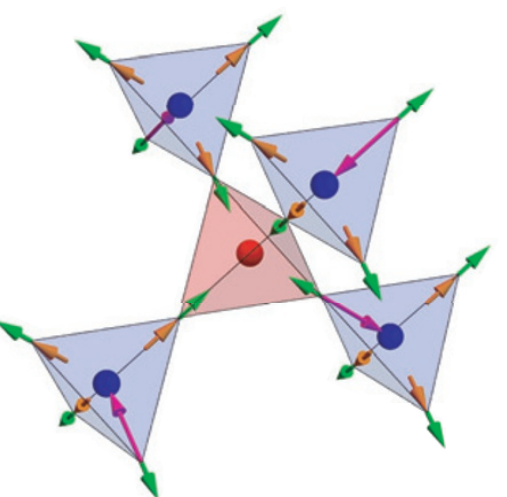

d

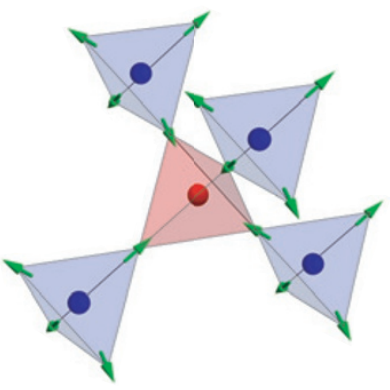

e

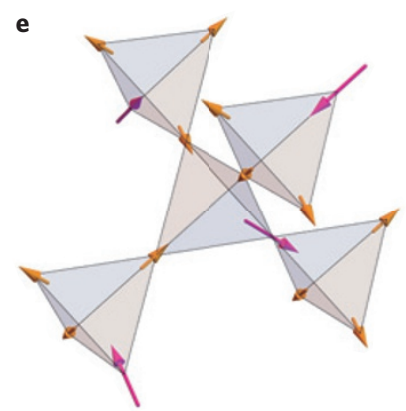

Figure 1 | Spin ice and magnetic fragmentation. $\mathbf{a}, \mathbf{b}$, Schematic of tetrahedra obeying the ice rule. $\mathbf{a}$, The spin state $\left\{s_{1}, s_{2}, s_{3}, s_{4}\right\}$ of a tetrahedron can be described using the convention: $s_{i}=1$ for a spin pointing in and -1 for a spin pointing out. The ice rule is simply written as $\sum_{i} s_{i}=0$, which corresponds to a divergence-free condition. b, A spin-flip generates two magnetic monopoles. The red (blue) monopole in $\mathbf{b}$ can be written $m_{+}=\{1,1,1,-1\}\left(m_{-}=\{-1,-1,-1,1\}\right)$, so that $\sum_{i} s_{i}= \pm 2$ on a tetrahedron. c-e, In the fragmentation theory, the local magnetic moment field fragments through a Helmholtz decomposition into two parts, a divergence-full and a divergence-free part. Each fragment carries components $s_{i} \neq \pm 1$. For instance, for the $m_{+}$monopole $\{1,1,1,-1\}$, this leads to: $\{1,1,1,-1\}=\{1 / 2,1 / 2,1 / 2,1 / 2\}+\{1 / 2,1 / 2,1 / 2,-(3 / 2)\}$. c Sketch of monopole crystallization, with the representation of the fragmented moments. d, The divergence-full contributions (green arrows)

$\{1 / 2,1 / 2,1 / 2,1 / 2\}$ and $\{-1 / 2,-1 / 2,-1 / 2,-1 / 2\}$, form an 'all in-all out' state and carry the magnetic charge $\left(\sum_{i} s_{i}= \pm 2\right)$. e, The second contribution is composed of three components $1 / 2$ (orange arrows) and one component $3 / 2$ (magenta) (for example $\{1 / 2,1 / 2,1 / 2,-(3 / 2)\}$ ), in such a way that the $\sum_{i} s_{i}=0$ constraint is fulfilled. It thus obeys a divergence-free condition, but is different from the ice rule, and forms a new kind of Coulomb phase. The same argument can be made for the other magnetic configurations $m_{+}=\{1,1,-1,1\},\{1,-1,1,1\}$, or $\{-1,1,1,1\}$ (and associated $m_{-}$). The divergence-full contribution remains identical, whereas the position of the magenta 3/2 component that emerges in the divergence-free contribution changes, as a fingerprint of the degeneracy.

of monopoles. If fragmentation occurs, the theory predicts that the magnetic structure factor should exhibit both Bragg peaks characteristic of the 'all in-all out' structure and a pinch point
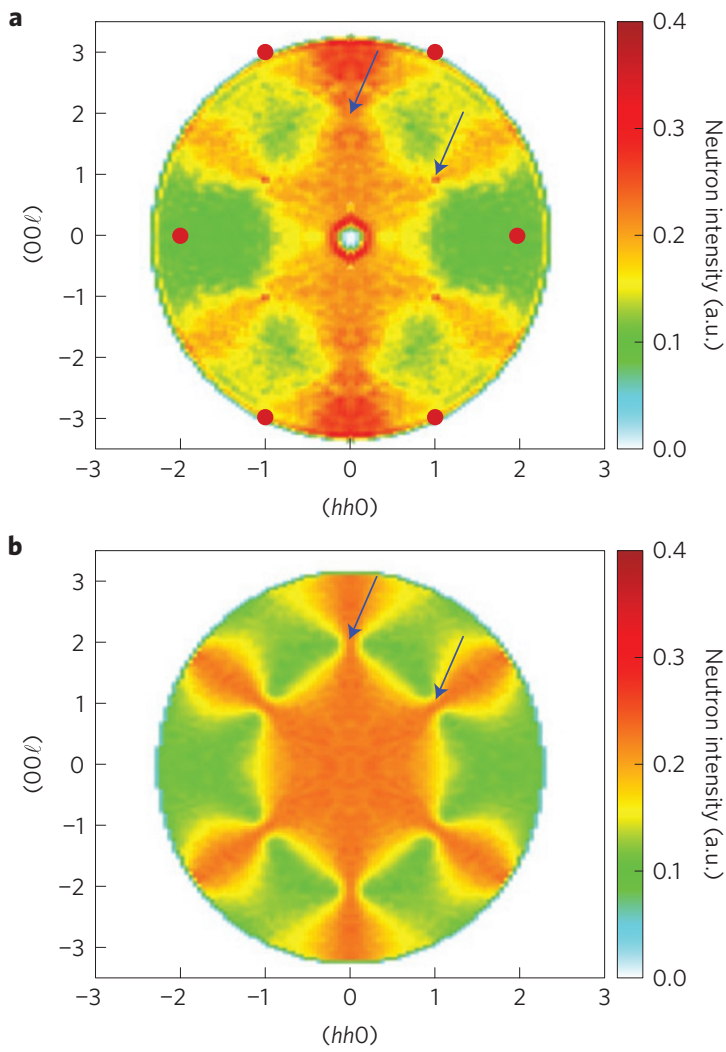

Figure $\mathbf{2}$ | Pinch point pattern in $\mathrm{Nd}_{2} \mathrm{Zr}_{2} \mathrm{O}_{7}$. a, Inelastic neutron scattering intensity averaged in the energy range $45<E<55 \mu \mathrm{eV}$ measured at $T=60 \mathrm{mK}$ with an incident wavelength $\lambda=6 \AA$. The red spots denote the antiferromagnetic Bragg peak positions that appear at zero energy transfer. b. Dynamical structure factor $S(Q, E=50 \mu \mathrm{eV})$ calculated in the RPA approximation for the pseudospin $1 / 2$ model described by equation (3), with $\mathcal{J}^{\prime}=1.2 \mathrm{~K}$ and $\mathcal{K}=-0.55 \mathrm{~K}$. The model takes into account a ferromagnetic exchange $\mathcal{J}^{\prime}$ along with a transverse interaction $\mathcal{K}$ whose physical origin is a coupling between octopoles. Blue arrows indicate the pinch point positions.

pattern typical of a Coulomb phase ${ }^{6}$. The pyrochlore system $\mathrm{Nd}_{2} \mathrm{Zr}_{2} \mathrm{O}_{7}$ is a good candidate in the search for such a system. Previous studies have provided evidence for the strong Ising character of the $\mathrm{Nd}^{3+}$ ion, and for ferromagnetic interactions, inferred from the positive Curie-Weiss temperature $\theta_{\mathrm{CW}}=195 \mathrm{mK}$ (ref. 16). Moreover, $\mathrm{Nd}_{2} \mathrm{Zr}_{2} \mathrm{O}_{7}$ orders below $T_{\mathrm{N}}=285 \mathrm{mK}$ in an 'all in-all out' state carrying a reduced ordered magnetic moment of about one third of the total $\mathrm{Nd}^{3+}$ magnetic moment $\mu_{\mathrm{eff}}=2.4 \mu_{\mathrm{B}}$ (ref. 17) (see Supplementary Information).

To demonstrate that the fragmentation occurs in $\mathrm{Nd}_{2} \mathrm{Zr}_{2} \mathrm{O}_{7}$, it is essential to observe signatures of the Coulomb phase. To this end, neutron scattering experiments have been carried out as a function of temperature and magnetic field on a large single crystal. As shown in Fig. 2a, the key point here is that the neutron data at $60 \mathrm{mK}$ do exhibit arm-like features along the $(00 \ell)$ and $(h h h)$ directions, with pinch points at the (002) and (111) positions, expected in the Coulomb phase ${ }^{6}$. This pinch point pattern is observed simultaneously with the 'all in-all out' Bragg peaks ${ }^{17,18}$ at (220) and (113), which we interpret as evidence for fragmentation and monopole crystallization.

Importantly, this structured neutron scattering signal appears as a flat mode at finite energy around $E_{\mathrm{o}}=70 \mu \mathrm{eV}$ (see Figs 3 top and $4 \mathrm{a}, \mathrm{b})$. In addition, above this flat mode, collective dispersive excitations stem from the pinch points and not from the antiferromagnetic 'all in-all out' wavevectors (see Fig. 3 top). They are characterized by a spin gap $\Delta \approx E_{\mathrm{o}}$ and reach a maximum energy of about $0.25 \mathrm{meV}$. 


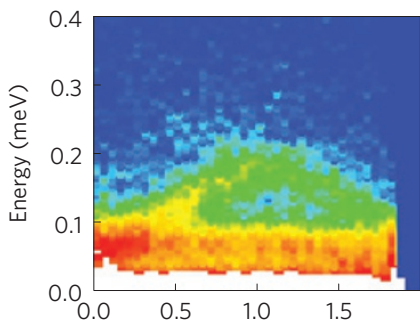

(hh2)

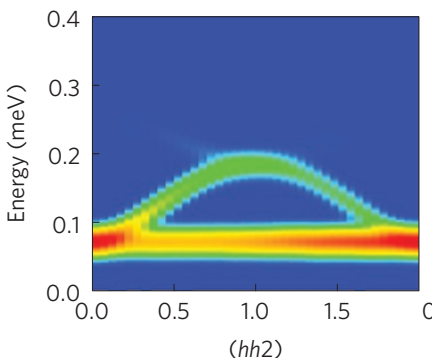

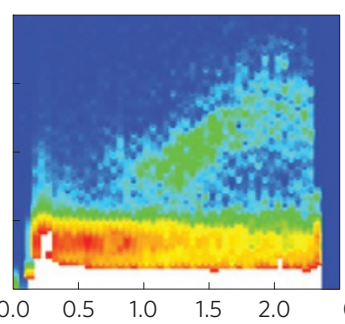

(hhO)

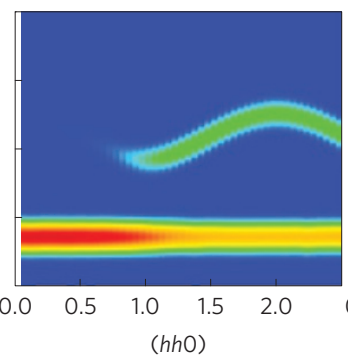

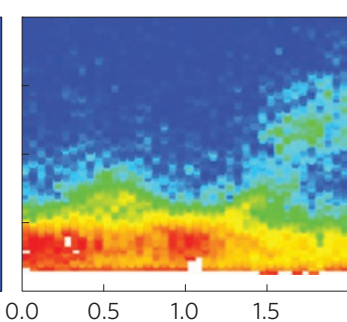

(hh 2-h)

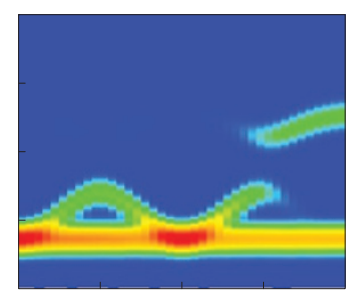

0.5

(hh 2-h)

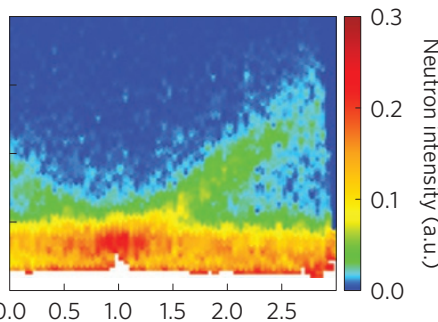

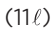

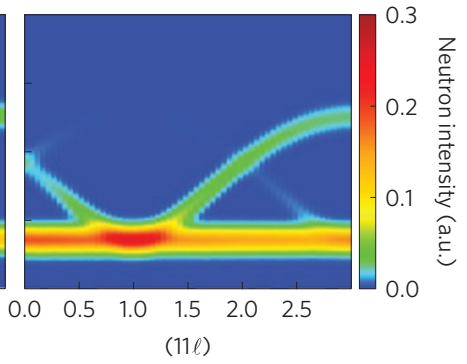

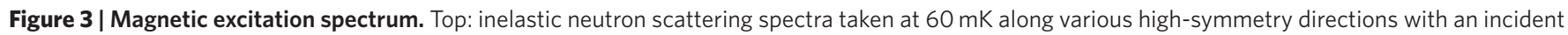
wavelength $\lambda=6 \AA$. Bottom: RPA calculation of the corresponding spectra for the pseudospin $1 / 2$ model described by equation ( 3 ), with $\mathcal{J}^{\prime}=1.2 \mathrm{~K}$ and $\mathcal{K}=-0.55 \mathrm{~K}$. Note that the same dispersion curve is obtained with a ferro octopolar coupling $\mathcal{K}=+1.65 \mathrm{~K}$ : the sign of the octopolar coupling does not

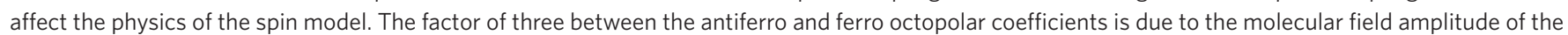
octopolar phase, the 'all in-all out' molecular field being three times larger than the 'two in-two out' field.

When increasing the temperature, the pinch point pattern and the collective modes persist up to $600 \mathrm{mK}$, far above the antiferromagnetic ordering $\left(T_{\mathrm{N}}=285 \mathrm{mK}\right)$ (see Supplementary Information). Whereas the energy gap, and the intensity of these features, decrease as the temperature increases (see Fig. 4a), the energy range of the dispersion remains unaffected up to $450 \mathrm{mK}$. This temperature dependence suggests a scenario in which the fragmentation takes place well above $T_{\mathrm{N}}$ : at the temperature where the ferromagnetic correlations start to develop, a Coulomb phase arises in coexistence with a liquid of monopoles. The latter finally crystallizes on cooling in an 'all in-all out' phase at $T_{\mathrm{N}}$, leaving the Coulomb phase unchanged. The field dependence is consistent with this scenario (see Fig. $4 \mathrm{~b}, \mathrm{c}$ ): at an applied field of $0.15 \mathrm{~T}$, where magnetization measurements show that the all inall out' state is replaced by a field-induced ordered state ${ }^{17}$, the Coulomb phase characteristics remain, albeit with less intensity. This observation further confirms the fragmentation scenario in which the divergence-free and divergence-full parts of the magnetic moment field behave independently.

This peculiar spin dynamics, and especially the existence of dispersive modes, are puzzling in an Ising-like system. They call for the existence of additional transverse terms in the Hamiltonian given in equation (2). To address this point, the magnetic moments should not be considered as Ising variables, but as pseudospin half $\sigma_{i}$ spanning the $|\uparrow \downarrow\rangle$ crystalline electric field (CEF) doublet states.

Considering the very peculiar 'dipolar-octopolar' nature of the Kramers $\mathrm{Nd}^{3+}$ doublet ${ }^{17,19-21}$, such transverse terms arise from a coupling between octopolar moments. Indeed, although $\langle\uparrow|\boldsymbol{J}| \downarrow\rangle \equiv 0$ because of those CEF properties, it can be shown using the explicit wavefunctions determined in ref. 17 that the octopole $\mathcal{T}=i\left(J^{+} J^{+} J^{+}-J^{-} J^{-} J^{-}\right)$is the relevant operator, because $\langle\uparrow|\mathcal{T}| \downarrow\rangle \neq 0$. Introducing an octopole-octopole coupling $\mathcal{V}=\sum_{\langle i, j} \kappa \mathcal{T}_{i} \mathcal{T}_{j}$, where $\kappa$ denotes the strength of the octopolar coupling, and projecting it onto the pseudospin $1 / 2$ subspace leads to:

$$
\mathcal{H}_{1 / 2}=\sum_{\langle i, j\rangle} \mathcal{J}^{\prime} \sigma_{i}^{z} \sigma_{j}^{z}+\mathcal{K} \sigma_{i}^{y} \sigma_{j}^{y}
$$

where $\sigma^{y, z}$ are the pseudospin components in the local coordinates, $\mathcal{J}^{\prime}$ is an effective exchange interaction $\mathcal{J}^{\prime}=\left(g_{z} / g_{\mathrm{I}}\right)^{2} \mathcal{J}_{\text {eff }}$ and $\mathcal{K}=4 \kappa|\langle\uparrow|\mathcal{T}| \downarrow\rangle|^{2}$. For $\mathrm{Nd}_{2} \mathrm{Zr}_{2} \mathrm{O}_{7}, g_{\mathrm{J}}=8 / 11, g_{x}=g_{y}=0$ and $g_{z}=4.5$.

The Hamiltonian parameters $\mathcal{J}^{\prime}$ and $\mathcal{K}$ can be estimated by fitting the inelastic neutron scattering spectra. From calculations

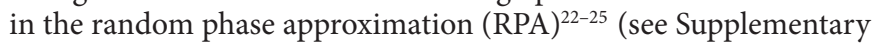
Information), it is found that the bandwidth of the collective modes is related to $\mathcal{J}^{\prime}$ whereas the shift of the pinch point pattern up to $E_{\mathrm{o}}$ is induced by the transverse term $\mathcal{K}$. This is reminiscent of the role of the antisymmetric Dzyaloshinskii-Moriya interaction which lifts the 'weathervane' flat mode in kagome systems up to finite energy ${ }^{26}$. Such transverse terms might also be at the origin of the inelastic pattern observed in the quantum spin ice candidate $\mathrm{Pr}_{2} \mathrm{Zr}_{2} \mathrm{O}_{7}$ (ref. 27). The best agreement is obtained for $\mathcal{J}^{\prime}=1.2 \mathrm{~K}$ and $\mathcal{K}=-0.55 \mathrm{~K}$ (see Figs $2 \mathrm{~b}$ and 3 bottom). For these values, the RPA ground state is an ordered octopolar phase. It is worth noting that, although this RPA calculation accounts for the behaviour of the divergence-free part of the magnetic moment, it is unable to capture the fragmentation mechanism.

We have thus shown that the predicted fragmentation process ${ }^{6}$ exists in the spin ice material $\mathrm{Nd}_{2} \mathrm{Zr}_{2} \mathrm{O}_{7}$. Below $700 \mathrm{mK}$, the magnetic moment field fragments into two parts: a divergencefull part which crystallizes at $T_{\mathrm{N}}=285 \mathrm{mK}$, and a divergencefree part for which transverse terms induce gapped and dispersive excitations. Our results highlight that the two fragments behave independently as a function of field and temperature, which opens the appealing possibility of manipulating them separately.

Beyond the classical fragmentation theory described in ref. 6, the importance of transverse terms to describe our observations emphasizes the need for considering quantum effects in further theoretical studies. Indeed, in the classical scheme, the crystallization occurs when the energy required to create the assembly of fragmented monopoles is balanced by the repulsive energy among them, and thus depends on the competition between exchange and dipolar interactions. In the present case, transverse octopolar couplings might enhance the interactions between monopoles, thus promoting their crystallization. We thus anticipate that our experiment will pave the way towards a quantum theory of fragmentation, involving such transverse terms. 

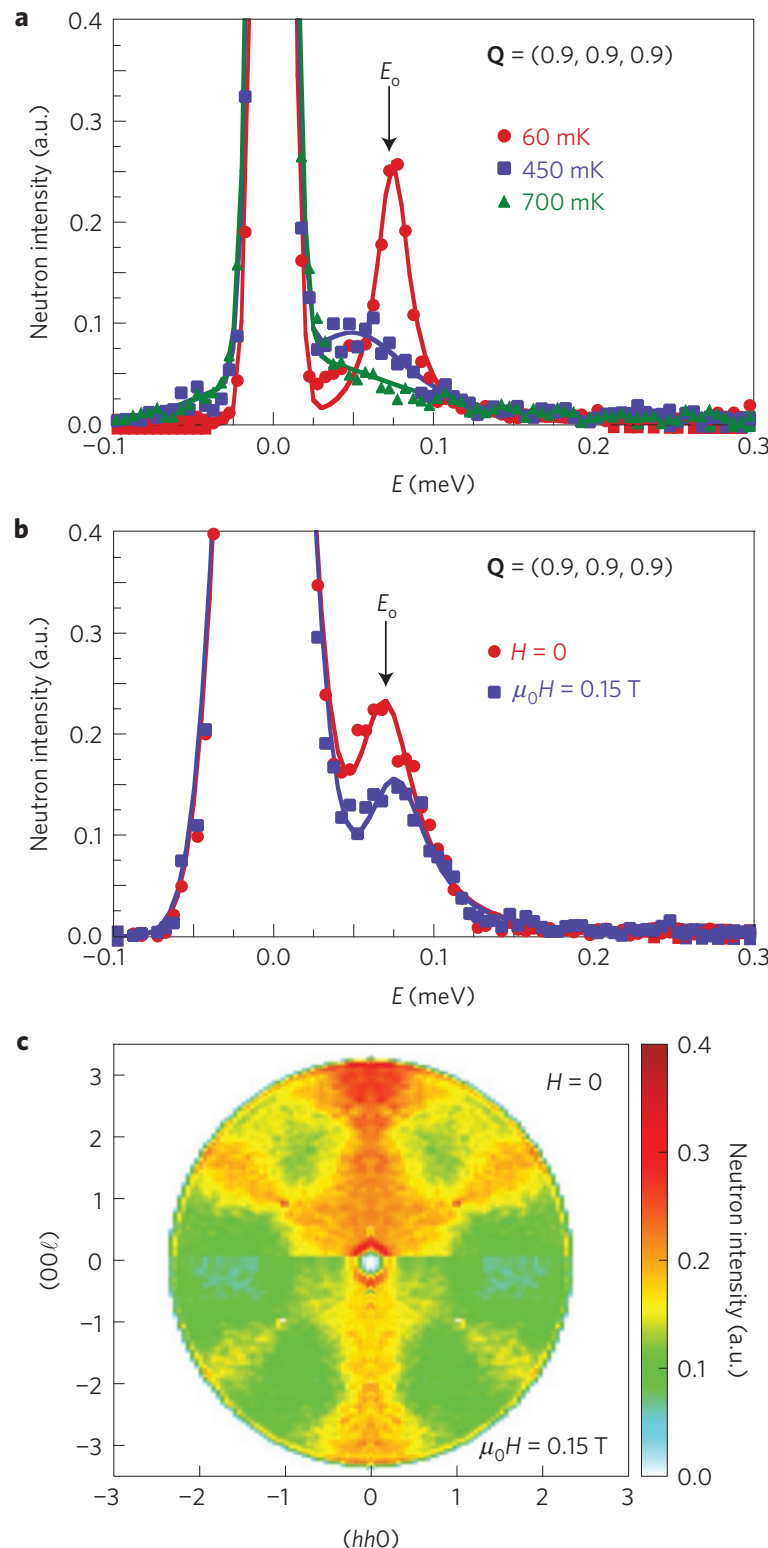

Figure 4 | Temperature and field dependence of the pinch point pattern. $\mathbf{a}, \mathbf{b}$, Excitation spectra at $\mathbf{Q}=(0.9,0.9,0.9)$ at several temperatures for $\lambda=8.5 \AA$ in zero field (a), and for $\lambda=6 \AA$ in zero field and in a $0.15 \mathrm{~T}$ applied field in the $[1-10]$ direction at $60 \mathrm{mK}(\mathbf{b})$. Above the strong elastic incoherent scattering, a flat mode appears at $E_{0}$, whose structure factor exhibits the pinch point pattern. Note that owing to the different energy resolution at $\lambda=6 \AA$, the peak appears broader in $\mathbf{b}$. $\mathbf{c}$, Inelastic neutron scattering map measured at $T=60 \mathrm{mK}$ and averaged in the energy range $45<E<55 \mu \mathrm{eV}$, showing a similar pattern but different intensity between zero field (top) and $0.15 \mathrm{~T}$ (bottom) data.

In a broader context, the fragmentation theory relies on the Helmholtz decomposition of a charged field, widely used to describe continuous fluid media in a wide variety of fields, from fluid mechanics to robotics ${ }^{28}$. This decomposition allows one to identify new relevant degrees of freedom, which could not have been separated otherwise. Our results in $\mathrm{Nd}_{2} \mathrm{Zr}_{2} \mathrm{O}_{7}$ indicate its applicability to describe, more generally, localized moment systems where fluctuating and ordered phases coexist. This might cover the case of the pyrochlore compound $\mathrm{Yb}_{2} \mathrm{Ti}_{2} \mathrm{O}_{7}$, a system showing a strongly reduced ferromagnetic ordering ${ }^{29}$ and a peculiar fluctuation spectrum, and whose physics is probably governed by competing phases ${ }^{25,30}$. In spin ice, the Helmholtz decomposition is applied at a microscopic level on the emergent gauge field of the Coulomb phase and on its charges, the monopoles. Our experimental findings give a concrete form to these concepts. The observation of fragmentation in $\mathrm{Nd}_{2} \mathrm{Zr}_{2} \mathrm{O}_{7}$ will thus stimulate new conceptual approaches in physical systems where such emergent fields exist.

\section{Methods}

Methods and any associated references are available in the online version of the paper.

Received 13 November 2015; accepted 25 February 2016; published online 4 April 2016

\section{References}

1. Stormer, H. L. Nobel lecture: the fractional quantum Hall effect. Rev. Mod. Phys. 71, 875-889 (1999).

2. Sachdev, S. Quantum Phase Transitions (Cambridge Univ. Press, 2011).

3. Bolotin, K. I., Ghahar, F., Shulman, M. D., Stormer, H. L. \& Kim, P. Observation of the fractional quantum Hall effect in graphene. Nature 462, 196-199 (2009).

4. Castelnovo, C., Moessner, R. \& Sondhi, S. L. Magnetic monopoles in spin ice. Nature 451, 42-45 (2008).

5. Henley, C. L. The Coulomb phase in frustrated systems. Annu. Rev. Condens. Matter. Phys. 1, 179-210 (2010).

6. Brooks-Bartlett, M. E., Banks, S. T., Jaubert, L. D. C., Harman-Clarke, A. \& Holdsworth, P. C. W. Magnetic-moment fragmentation and monopole crystallization. Phys. Rev. X 4, 011007 (2014).

7. den Hertog, B. C. \& Gingras, M. J. P. Dipolar interactions and origin of spin ice in Ising pyrochlore magnets. Phys. Rev. Lett. 84, 3430-3433 (2000).

8. Harris, M. J., Bramwell, S. T., McMorrow, D. F., Zeiske, T. \& Godfrey, K. W. Geometrical frustration in the ferromagnetic pyrochlore $\mathrm{Ho}_{2} \mathrm{Ti}_{2} \mathrm{O}_{7}$. Phys. Rev. Lett. 79, 2554-2557 (1997).

9. Ramirez, A. P., Hayashi, A., Cava, R. J., Siddharthan, R. \& Shastry, B. S. Zero-point entropy in spin ice. Nature 399, 333-335 (1999).

10. Huse, D. A., Krauth, W., Moessner, R. \& Sondhi, S. L. Coulomb and liquid dimer models in three dimensions. Phys. Rev. Lett. 91, 167004 (2003).

11. Bergman, D. L., Fiete, G. A. \& Balents, L. Ordering in a frustrated pyrochlore antiferromagnet proximate to a spin liquid. Phys. Rev. B 73, 134402 (2006).

12. Isakov, S. V., Gregor, K., Moessner, R. \& Sondhi, S. L. Dipolar spin correlations in classical pyrochlore magnets. Phys. Rev. Lett. 93, 167204 (2004).

13. Henley, C. L. Power-law spin correlations in pyrochlore antiferromagnets. Phys. Rev. B 71, 014424 (2005)

14. Fennell, T. et al. Magnetic Coulomb phase in the spin ice $\mathrm{Ho}_{2} \mathrm{Ti}_{2} \mathrm{O}_{7}$. Science 326, 415-417 (2009).

15. Morris, D. J. P. et al. Dirac strings and magnetic monopoles in the spin ice $\mathrm{Dy}_{2} \mathrm{Ti}_{2} \mathrm{O}_{7}$. Science 326, 411-414 (2009).

16. Ciomaga Hatnean, M. et al. Structural and magnetic investigations of single-crystalline neodymium zirconate pyrochlore $\mathrm{Nd}_{2} \mathrm{Zr}_{2} \mathrm{O}_{7}$. Phys. Rev. B 91, 174416 (2015).

17. Lhotel, E. et al. Fluctuations and all-in-all-out ordering in dipole-octopole $\mathrm{Nd}_{2} \mathrm{Zr}_{2} \mathrm{O}_{7}$. Phys. Rev. Lett. 115, 197202 (2015).

18. Ferey, G., de Pape, R., Leblanc, M. \& Pannetier, J. Ordered magnetic frustration: VIII. Crystal and magnetic structures of the pyrochlore form of $\mathrm{FeF}_{3}$ between 2.5 and $25 \mathrm{~K}$ from powder neutron diffraction. Comparison with the other varieties of $\mathrm{FeF}_{3}$. Rev. Chim. Miner. 23, 474-484 (1986).

19. Abragam, A. \& Bleaney, B. Electron Paramagnetic Resonance of Transition Ions (Oxford Classic Texts in the Physical Sciences, Oxford Univ. Press, 1970).

20. Watahiki, M. et al. Crystalline electric field study in the pyrochlore $\mathrm{Nd}_{2} \operatorname{Ir}_{2} \mathrm{O}_{7}$ with metal-insulator transition. J. Phys. Conf. Ser. 320, 012080 (2011).

21. Huang, Y.-P., Chen, G. \& Hermele, M. Quantum spin ices and topological phases from dipolar-octupolar doublets on the pyrochlore lattice. Phys. Rev. Lett. 112, 167203 (2014).

22. Jensen, J. \& Mackintosh, A. R. Rare Earth Magnetism (International Series of Monographs on Physics, Clarendon, 1991).

23. Kao, Y. J., Enjalran, M., Del Maestro, A., Molavian, H. R. \& Gingras, M. J. P. Understanding paramagnetic spin correlations in the spin-liquid pyrochlore $\mathrm{Tb}_{2} \mathrm{Ti}_{2} \mathrm{O}_{7}$. Phys. Rev. B 68, 172407 (2003).

24. Petit, S. et al. Order by disorder or energetic selection of the ground state in the $\mathrm{XY}$ pyrochlore antiferromagnet $\mathrm{Er}_{2} \mathrm{Ti}_{2} \mathrm{O}_{7}$. An inelastic neutron scattering study. Phys. Rev. B 90, 060410 (2014).

25. Robert, J. et al. Spin dynamics in the presence of competing ferromagnetic and antiferromagnetic correlations in $\mathrm{Yb}_{2} \mathrm{Ti}_{2} \mathrm{O}_{7}$. Phys. Rev. B 92, 064425 (2014).

26. Matan, K. et al. Spin Waves in the Frustrated Kagomé Lattice Antiferromagnet $\mathrm{KFe}_{3}(\mathrm{OH})_{6}\left(\mathrm{SO}_{4}\right)_{2}$. Phys. Rev. Lett. 96, 247201 (2006). 
27. Kimura, K. et al. Quantum fluctuations in spin-ice-like $\operatorname{Pr}_{2} \mathrm{Zr}_{2} \mathrm{O}_{7}$. Nature Commun. 4, 1934 (2013).

28. Bhatia, H., Norgard, G., Pascucci, V. \& Bremer, P.-T. The Helmholtz-Hodge decomposition-a survey. IEEE Trans. Vis. Comput. Graphics 19, 1386-1404 (2013).

29. Chang, L.-J. et al. Higgs transition from a magnetic Coulomb liquid to a ferromagnet in $\mathrm{Yb}_{2} \mathrm{Ti}_{2} \mathrm{O}_{7}$. Nature Commun. 3, 992 (2012).

30. Jaubert, L. D. C. et al. Are multiphase competition and order by disorder the keys to understanding $\mathrm{Yb}_{2} \mathrm{Ti}_{2} \mathrm{O}_{7}$ ? Phys. Rev. Lett. 115, 267208 (2015).

\section{Acknowledgements}

We acknowledge the ILL for the beam time. We also thank J. Robert,

P. C. W. Holdsworth, V. Simonet and Y. Sidis for fruitful discussions. M.C.H., M.R.L. and

G.B. acknowledge financial support from the EPSRC, UK, Grant No. EP/M028771/1.

\section{Author contributions}

Crystal growth and characterization were performed by M.C.H., M.R.L. and G.B. Inelastic neutron scattering experiments were carried out by S.P., E.L., J.O. and H.M. Diffraction experiments were carried out by S.P., E.L., A.R.W. and E.R. The data were analysed by S.P. and E.L., with input from B.C., A.R.W., E.R. and J.O. RPA calculations were carried out by S.P. The paper was written by E.L. and S.P., with feedback from all authors.

\section{Additional information}

Supplementary information is available in the online version of the paper. Reprints and permissions information is available online at www.nature.com/reprints.

Correspondence and requests for materials should be addressed to S.P. or E.L.

\section{Competing financial interests}

The authors declare no competing financial interests. 


\section{Methods}

Single crystals of $\mathrm{Nd}_{2} \mathrm{Zr}_{2} \mathrm{O}_{7}$ were grown by the floating-zone technique using a four-mirror xenon arc lamp optical image furnace ${ }^{16,31}$.

Inelastic neutron scattering experiments were carried out at the Institute Laue Langevin (ILL, France) on the IN5 disk chopper time-of-flight spectrometer operated at $\lambda=8.5 \AA$ or $\lambda=6 \AA$. The $\mathrm{Nd}_{2} \mathrm{Zr}_{2} \mathrm{O}_{7}$ single-crystal sample was attached to the cold finger of a dilution insert and the field was applied along $\left[\begin{array}{lll}1 & -1\end{array}\right]$. The data were processed with the Horace software, transforming the recorded time of flight, sample rotation and scattering angle into energy transfer and Q-wavevectors.

The neutron diffraction data were taken at the D23 single-crystal diffractometer (CEA-CRG, ILL France) with a copper monochromator and using $\lambda=1.28 \AA$. Here the field was applied along the [111] direction. Refinements were carried out with the Fullprof software suite ${ }^{32}$ (http://www.ill.eu/sites/fullprof).

The magnetic diffuse scattering was measured on the D7 diffractometer installed at the ILL, with $\lambda=4.85 \AA$, using a standard polarization analysis technique with the guiding field along the vertical axis $\left[\begin{array}{lll}1 & -1 & 0\end{array}\right]$.
Calculations are carried out on the basis of a mean field treatment of a Hamiltonian, taking into account the dipolar exchange as well as an octopolar coupling between the crystalline electric field (CEF) ground doublet states of the $\mathrm{Nd}^{3+}$ ion. This Hamiltonian is written in terms of a pseudospin $1 / 2$ spanning these states. The spin dynamics is then calculated numerically in the random phase approximation ${ }^{22-25}$.

More details are provided in the Supplementary Information.

\section{References}

31. Ciomaga Hatnean, M., Lees, M. R. \& Balakrishnan, G. Growth of single-crystals of rare-earth zirconate pyrochlores, $\mathrm{Ln}_{2} \mathrm{Zr}_{2} \mathrm{O}_{7}$ (with $\mathrm{Ln}=\mathrm{La}$, $\mathrm{Nd}, \mathrm{Sm}$, and $\mathrm{Gd}$ ) by the floating zone technique. J. Cryst. Growth 418, 1-6 (2015).

32. Rodríguez-Carvajal, J. Recent advances in magnetic structure determination by neutron powder diffraction. Physica B 192, 55-69 (1993). 